\title{
The Human Plasma Lipidome
}

\author{
Oswald Quehenberger, Ph.D. and Edward A. Dennis, Ph.D. \\ Departments of Medicine and Pharmacology (O.Q.) and the Departments of Chemistry and \\ Biochemistry and Pharmacology (E.A.D.), School of Medicine, University of California, San \\ Diego, La Jolla.
}

\begin{abstract}
CHOLESTEROL AND TRIGLYCERIDE LEVELS ARE NOW ASSESSED
ROUTINELY, and physicians regularly prescribe lipid-lowering drugs to patients found to have dyslipidemia. However, the increase in the number of patients with metabolic diseases (including type 2 diabetes and obesity, which are associated with an elevated risk of cardiovascular disease) demands more detailed lipid analyses both for diagnostic purposes and for monitoring the efficacy of prescribed therapy. Human plasma comprises nucleic acids, amino acids (mostly in the form of proteins), carbohydrates (in the form of monosaccharides and disaccharides), and lipids (Fig. 1). ${ }^{1-3}$ Much is known about the first three constituents, but among the various cellular metabolites, lipids stand out because of their structural diversity and the sheer number of discrete molecular species - in the hundreds of thousands, according to some estimates ${ }^{4}$ (Fig. 2).
\end{abstract}

The lipids in plasma are solubilized and dispersed through their association with specific groups of proteins. Most free fatty acids and related structures with carboxyl functional groups associate with albumin, whereas the transport and distribution of more complex lipids are accomplished by means of plasma lipoproteins. ${ }^{5}$ The structural diversity of lipids is mirrored by the enormous variation in their physiological function. The abundance of individual lipid molecular species in plasma may be indicative of the variety of specific human diseases. In this review, we discuss the application of metabolic-profiling strategies to quantitatively measure entire lipid categories in human plasma, and we examine the potential use of the diverse lipid metabolites as diagnostic tools or therapeutic targets.

\section{DIVERSITY OF LIPIDS IN HUMAN PLASMA}

In view of the importance of lipids to biologic and pathophysiological processes, detailed knowledge of the composition and concentration of lipid metabolites in plasma would be expected to expand our diagnostic capabilities. To this end, the National Institute of Diabetes and Digestive and Kidney Diseases (NIDDK), in collaboration with the National Institute of Standards and Technology (NIST), recently issued a plasma-based standard reference material (SRM 1950 Metabolites in Human Plasma) for the purpose of metabolite analysis (www.nist.gov). Historically, wide-scale lipid profiling has been difficult to perform because the diverse physical properties of lipid metabolites require a multitude of purification systems combined with a host of complex technical procedures. The evolution of lipidomics has driven the development of new analytic platforms, specifically in the area of mass spectrometry, which have streamlined these procedures and have allowed many more lipid molecules to be analyzed in great detail. A high-resolution lipidomic method

Copyright () 2011 Massachusetts Medical Society. All rights reserved.

Address reprint requests to Dr. Dennis at the University of California, San Diego, School of Medicine, Department of Pharmacology, 9500 Gilman Dr., La Jolla, CA 92093-0601, or at edennis@ucsd.edu..

Disclosure forms provided by the authors are available with the full text of this article at NEJM.org. 
based on liquid and gas chromatography coupled with mass spectrometry and designed to systematically map the mammalian lipidome was applied to the NIDDK-NIST SRM, facilitating the first comprehensive lipid analysis of human plasma. ${ }^{3}$ The in-depth analysis performed by the LIPID MAPS consortium (www.lipidmaps.org) revealed an amazingly complex plasma lipidome. Almost 600 distinct molecular species were quantified, covering the six main mammalian lipid categories: fatty acyls, glycerolipids, glycerophospholipids, sphingolipids, sterol lipids, and prenol lipids ${ }^{6}$ (Fig. 3). Their structural diversity is particularly apparent within the sphingolipid and glycerophospholipid categories, with 204 and 160 distinctly identifiable molecular species, respectively. The somewhat lower number of defined species in the glycerolipid category is largely due to the presence of complex mixtures of isobaric entities that make it difficult to define individual molecular species. The heterogeneity of complex lipids within a category is principally determined by variations in fatty acid content and head groups. The fatty acids used in the assembly of complex lipids come from several sources, including nutrient intake, release from adipose tissue, and biosynthesis.

\section{PLASMA LIPIDS IN THE METABOLIC SYNDROME OBESITY AND GLYCEROLIPIDS}

Glycerolipids account for a high proportion of total lipids in plasma and comprise triacylglycerols (TAGs), diacylglycerols (DAGs), and ether-linked glycerolipids. The absolute plasma concentration of TAGs, distributed between chylomicrons and very-lowdensity lipoprotein (VLDL), is dependent on food intake (Fig. 4). In addition, several acquired or secondary factors (including genetic determinants, uncontrolled diabetes mellitus, obesity, and sedentary lifestyle) can cause hypertriglyceridemia, a prevalent form of dyslipidemia that is frequently associated with premature coronary artery disease. ${ }^{7}$ Public surveys have revealed that the prevalence of hypertriglyceridemia among adults 20 years of age or older in the United States is approximately $36 \%$ for men and $27 \%$ for women. ${ }^{8}$ On detailed lipidomic analyses performed independently in 14 pairs of young-adult monozygotic twins who were discordant for obesity ${ }^{9}$ and in a pool of 100 healthy adults, ${ }^{3}$ a considerable number of individual glycerolipid molecular structures were implicated, including TAGs and DAGs. In these studies, TAG 50:2, 52:2, 52:3, and 52:4 were the most abundant glycerolipid species. (TAGS are defined as the ratio of the total number of carbon atoms to the total number of double bonds in the fatty acids.) When the twin study was controlled for patientage and genetic background, it showed that several (but not all) TAG molecular species, including 56:4, correlated significantly with bodymass index and measurements of subcutaneous fat and may serve as biomarkers for the early detection of acquired obesity, especially when applied to children. ${ }^{9}$ Similarly, plasma profiling of 16 adults without diabetes with a broad range of insulin sensitivity revealed that TAG species containing saturated or monounsaturated fatty acids correlate positively with insulin resistance, whereas TAGs containing essential fatty acids correlate negatively with insulin resistance (according to the homeostasis model assessment) and waist circumference. ${ }^{10}$

The study of monozygotic twins included an assessment of phospholipids and showed that obesity was associated with increases in lysoglycerophosphocholine and decreases in ether glycerophospholipids. ${ }^{9}$ In addition, a strong negative correlation was seen between docosahexaenoic acid (DHA 22:6n-3)-containing glycerophosphocholine and levels of subcutaneous and intraabdominal fat. A separate lipidomic analysis of 19 hypertensive persons and 59 normotensive control subjects showed that hypertension was also associated with a decrease in ether glycerophospholipids - specifically, the ones containing arachidonic acid (20:4 n-6) and docosapentaenoic acid (DPA 22:5n-3). ${ }^{11}$ 
In a study of patients with dilated cardiomyopathy, researchers identified specific plasma lipid abnormalities; the predominant abnormality was a decrease in TAG molecular species containing odd-chain-length fatty acids. ${ }^{12}$ No marked changes in total triglycerides were observed; consequently, these specific molecular changes would remain undetected if one were using the enzymatic triglyceride assays that are routinely applied in clinical laboratories.

\section{SATURATED AND UNSATURATED FATTY ACIDS IN THE REGULATION OF ENERGY METABOLISM}

The contribution of lipids to our energy supply is quantitatively important, especially during prolonged muscular exercise, when as much as $80 \%$ of the energy is supplied by lipid oxidation. ${ }^{13}$ However, when dietary lipid intake exceeds energy expenditure, adipose lipid storage expands in the form of TAGs and results in overweight and obesity. To achieve and maintain a favorable body weight, emphasis is commonly placed on the amount of fat in the diet, whereas lipid composition is frequently ignored. However, the fatty acid composition of fats ingested may determine lipid energy substrate utilization in humans. Since the 1960s, the intake of calories in the form of fat has steadily decreased, yet during the same period, obesity has increased in the U.S. population. ${ }^{14,15}$ The molecular structure of the fatty acids determines the kinetics of their release from adipose tissue. The mobilization of fatty acids increases with the degree to which they are unsaturated, and as predicted, arachidonic acid, eicosapentaenoic acid (EPA 20:5 n-3), and DHA, the latter two being major components of fish oil, are released at high rates relative to the more saturated fatty acids. ${ }^{16}$ Consistent with this process is the finding that unsaturated fatty acids undergo more rapid oxidation than do their saturated analogues, suggesting that the composition of fat depots may be as important as their total quantity. ${ }^{17}$

Most obese persons have elevated plasma levels of free fatty acids, owing to increased release from expanded adipose tissue. ${ }^{18}$ Long-term increases in levels of plasma free fatty acids will ultimately inhibit the antilipolytic action of insulin, which will further increase the rate of their release into the circulation and contribute to the development of insulin resistance and type 2 diabetes (Fig. 4). ${ }^{19}$ The plasma pool of free fatty acids is also an important source of lipid for hepatocytes, and any excess of free fatty acids is likely to be converted to TAGs and stored in the liver or incorporated into lipoproteins and secreted into the circulatory system. ${ }^{20}$ Predictably, raising plasma free fatty acid levels will lead to an accumulation of TAGs in the liver, which not only triggers hepatic insulin resistance but may also cause hepatic steatosis. ${ }^{19,21}$ Thus, specific free fatty acids are responsible for several serious health problems associated with obesity. ${ }^{22}$

\section{FREE FATTY ACID SIGNALING AND INSULIN SENSITIVITY}

Fatty acids have broad metabolic functions, owing to their ability to serve as potent signaling molecules. As described above, long-term elevations of plasma free fatty acid levels lead to insulin resistance in muscle, desensitization of adipocytes to the lipogenic effects of insulin, and steatosis in the liver (Fig. 4). ${ }^{19,23}$ Thus, plasma free fatty acids provide a metabolic link among obesity, insulin resistance, and type 2 diabetes - all wellestablished risk factors for cardiovascular disease. ${ }^{24}$ However, not all types of fatty acids contribute equally to the systemic disruption of insulin action. Epidemiologic studies indicate that fat rich in saturated fatty acids promotes insulin resistance, whereas monounsaturated and polyunsaturated fatty acids reduce it. ${ }^{25}$

A mouse model of obesity-induced insulin resistance showed that hepatic fatty acid composition was an independent determinant of insulin resistance. ${ }^{26}$ Modification of this composition by genetic inhibition of the elongation of palmitic acid (16:0) to stearic acid 
(18:0) ameliorated insulin resistance, even in the presence of persistent obesity and hepatosteatosis. Inhibition of elongation leads to a relative enrichment in palmitic acid, which can then serve as a substrate for desaturases to form monounsaturated palmitoleic acid (16:1). Studies of mice with a deficiency of the fatty acid-binding proteins aP2 and mal1 (FABP4 and FABP5, respectively) raised the possibility that local alterations in adipose tissue might regulate systemic metabolic responses. ${ }^{27}$ Although these mice were protected from the onset of diet-induced insulin resistance and fatty liver disease, total plasma free fatty acid levels paradoxically rose significantly. Targeted lipidomic analysis showed a unique lipid profile with significant enrichment of endogenous palmitoleate in adipose tissue and plasma, which led to the hypothesis that palmitoleate levels are associated with an improved metabolic profile. ${ }^{28}$ Consistent with this idea is the finding that palmitoleate stimulates basal glucose uptake and use by cultured skeletal-muscle cells. ${ }^{29} \mathrm{As}$ compared with the findings in animal models, the results of clinical studies are less clear. One recent study suggested a strong association between circulating levels of palmitoleate and insulin sensitivity, ${ }^{30}$ whereas another showed no correlation. ${ }^{31}$ However, the latter group of investigators found a robust association between plasma levels of transpalmitoleate and insulin sensitivity. ${ }^{32}$ The divergent findings for cis-palmitoleate and trans-palmitoleate remain unexplained, but the benefits derived from the trans isomer are supported by the clinical observation that the consumption of dairy products rich in trans-palmitoleate inversely correlates with the insulin resistance syndrome. ${ }^{33}$

\section{FISH OIL, EICOSANOIDS, AND INFLAMMATION}

Plasma free fatty acids have also been linked to sudden cardiac death, ${ }^{34}$ and increased levels have been observed in cancer-induced cachexia and may impair lymphocyte function. ${ }^{35,36}$ Several studies in animals and humans have shown that saturated and monounsaturated fatty acids increase the risk of ventricular arrhythmia and sudden cardiac death, whereas polyunsaturated fatty acids, and $n-3$ fatty acids in particular, prevent this arrhythmogenic action, with a significant reduction in the risk of death from cardiac causes. ${ }^{37,38}$ Presumably, polyunsaturated fatty acids act through their eicosanoid products, including prostaglandins, leukotrienes, and other oxidized fatty acid products (Fig. 4). ${ }^{39}$ The observed beneficial effects prompted the American Heart Association to release a statement regarding the nutritional benefits of $\mathrm{n}-3$ fatty acid consumption. ${ }^{40}$

Chronic inflammation in liver and adipose tissue is common in obesity, and mounting evidence indicates that free fatty acids directly modulate inflammatory responses. Several groups have characterized orphan G-protein-coupled receptors (GPR) that are specifically activated by free fatty acids. ${ }^{41,42} \mathrm{~A}$ number of these receptors are expressed at high levels on leukocytes and adipose tissue and may synchronize the inflammatory action of lipopolysaccharides. Whereas saturated fatty acids amplify the proinflammatory action of lipopolysaccharides, many polyunsaturated fatty acids have antiinflammatory effects. Consistent with these findings is the observation that $\mathrm{n}-3$ fatty acids, including DHA, enhance systemic insulin sensitivity in animal models of obesity by ameliorating inflammation in adipose tissue through a receptor termed GPR $120 .{ }^{42}$ These findings indicate the presence of interconnecting pathways linking fatty acid metabolism to inflammation. In addition, the $\mathrm{n}-3$ fatty acids have been implicated in brain function; a decrease in DHA and increased ratios of $n-6$ to $n-3$ polyunsaturated fatty acids in the serum predict the severity of some neurologic disorders including Alzheimer's disease and other dementia disorders, ${ }^{43,44}$ major depression, ${ }^{45}$ and bipolar affective disorder. ${ }^{46}$ Furthermore, a general populationbased study showed that several lipid clusters containing mainly saturated TAGs were significantly associated with schizophrenia. ${ }^{47}$ 


\section{CHOLESTEROL, OXIDIZED STEROLS, AND CARDIOVASCULAR RISK}

Cholesterol is the most abundant sterol in plasma and exists in both free and fatty acylesterified forms. In plasma, cholesterol is associated with lipoproteins, including mainly low-density lipoprotein (LDL, which accounts for 60 to $70 \%$ of plasma cholesterol) and high-density lipoprotein (HDL, which accounts for 20 to $30 \%$ of plasma cholesterol), and its level in plasma is widely used to predict a person's risk of cardiovascular events. In fact, a high level of LDL cholesterol in the blood is considered the major risk factor for coronary artery disease and stroke, making it a prominent target for therapeutic intervention. Current public health guidelines recommend that all persons 20 years of age or older be tested every 5 years to determine their fasting lipoprotein profile. ${ }^{48}$

Typical blood tests include measurement of total cholesterol, usually performed without discrimination between free and esterified forms. Cholesterol exists primarily in the form of fatty acyl esters; plasma contains more than 22 distinct molecular species of cholesterol ester. ${ }^{3}$ In addition to cholesterol, human plasma contains campesterol and sitosterol, which are not synthesized in humans but are derived entirely from the diet. In normal persons, plant sterols are present in only small amounts, and cholesterol accounts for more than $99 \%$ of all circulating sterols. In sitosterolemia, an autosomal recessive disorder, the levels of plant sterols in plasma are markedly elevated. ${ }^{49}$ In patients with this disorder, plasma cholesterol levels are also elevated, and xanthomatosis and premature atherosclerosis develop. ${ }^{50,51}$ These patients have a poor response to statin treatment but have a strikingly good response to treatment with bile acid resins. ${ }^{52} \mathrm{~A}$ complete plasma sterol panel is therefore necessary to determine the differential diagnosis and select the optimal treatment.

Lathosterol is also present in normal plasma and can be of diagnostic value as an indicator of whole-body cholesterol synthesis. ${ }^{53}$ The rate at which cholesterol is synthesized and the differentiation of this endogenous source from dietary contributions are of considerable clinical interest, especially in assessing the efficacy of statin therapy in patients with hypercholesterolemia. Normal plasma also contains trace amounts of 25-hydroxycholesterol, which is secreted by macrophages in response to the activation of toll-like receptors. ${ }^{54}$ Exposure of B cells to this macrophagederived oxysterol suppresses immunoglobulin A production, thereby facilitating negative regulation of the adaptive immune response by the innate immune system.

\section{PHOSPHOLIPIDS AND SPHINGOLIPIDS IN CANCER AND NEUROLOGIC DISEASES}

Glycerophospholipids constitute the main components of cell membranes and serve as precursors for signaling molecules in many cellular and physiological processes. As shown in Figures 2 and 3, phospholipids in plasma are abundant and heterogeneous, properties that are largely a function of fatty acid variations as well as structural differences of the head groups. Most phospholipids found in plasma are secreted by the liver and distributed among all lipoprotein classes..$^{5}$ Their structural diversity suggests critical involvement in many physiological and pathophysiological processes. ${ }^{3}$ Cancer cells have aberrant glycerophosphocholine metabolism, leading to an elevation of phosphocholine, an intermediate in glycerophosphocholine biosynthesis, and other cholinecontaining phospholipids. ${ }^{55,56}$ Elevated phosphocholine levels have been reported in several types of cancers and have been evaluated as a target for anticancer therapy. ${ }^{57-59}$ Moreover, phosphocholine levels may also be used as a predictor of both outcome and the aggressiveness of carcinomas ${ }^{60,61}$ and as an early marker of the response to therapy. ${ }^{62-64}$ 
In addition to a direct role in cancer biology, glycerophosphocholine serves as a precursor for several lipid second messengers, including phosphatidic acid, a signaling molecule implicated in the development of drug resistance that is frequently observed in rapamycin (sirolimus)-based immunosuppressive therapy and anticancer therapy. ${ }^{65}$ Phosphatidic acid can be further metabolized to lysophosphatidic acid by phospholipases $\mathrm{A}_{1}$ and $\mathrm{A}_{2}$, which hydrolyze the fatty acid at the sn- 1 position and the sn- 2 position, respectively. ${ }^{66}$ Lysophosphatidic acid is also formed outside cells in plasma from lysoglycerophosphocholine by autotaxin, which has lysophospholipase D activity and cleaves the choline head group from lysoglycerophosphocholine.$^{67}$ Lysophosphatidic acid is a bioactive lipid that signals through specific GPR to elicit a host of cellular responses, including proliferation, survival, and migration. ${ }^{68}$ Enhanced autotaxin expression and overproduction of lysophosphatidic acid have been noted in numerous types of cancer, including ovarian cancer. ${ }^{69}$ Ascites fluid from patients with ovarian cancer contains lysophosphatidic acid in high levels, ${ }^{70,71}$ an observation that is consistent with its role in tumor biology. Lysophosphatidic acid levels are also elevated in plasma from patients with ovarian cancer but not in plasma from healthy controls ${ }^{72}$; however, a second study could not verify these findings. ${ }^{73}$ Subsequent studies supported the potential role of plasma lysophosphatidic acid as a biomarker for ovarian cancer. ${ }^{74-77}$ To date, this issue remains controversial and is the impetus for an ongoing clinical trial (ClinicalTrials.gov number, NCT00986206).

Sphingolipids are complex lipids that are particularly abundant in nervous tissue. They are also found in human plasma, which contains over 200 distinct molecular species. ${ }^{3}$ Among these, the free sphingoid base sphingosine-1-phosphate (S1P) has potent messenger functions and is centrally involved in the trafficking of immune cells, maintenance of vascular tone, and cell communication in the central nervous system. ${ }^{78}$ It is detected in various tissues and blood and participates in numerous fundamental biologic and pathophysiological processes through five GPR S1P subtypes (S1P1 through S1P5) ${ }^{79}$ S1P regulates lymphocyte trafficking from the thymus and lymphoid organs, which requires the engagement of the S1P1receptor subtype on lymphocytes. ${ }^{80}$ Pharmacologic downmodulation of S1P1 antagonizes the action of S1P and prevents the egress of lymphocytes from the lymph node into the lymphatic circulation. This has implications for certain autoimmune diseases characterized by autoreactive lymphocytes and has led to the development of the immunosuppressive drug fingolimod (FTY720) for the treatment of multiple sclerosis. ${ }^{81}$ Fingolimod, an S1P analogue, down-modulates four of the five S1P receptors, creating temporary S1P1-null lymphocytes and thus favors their retention in lymph nodes.

Sphingolipids are also implicated in a number of neurologic diseases. Recent profiling efforts established baseline levels for more than 200 sphingolipids in unfractionated plasma and across lipoprotein fractions isolated from healthy donors. $3,82,83$ Plasma levels of glucosylceramide and ceramide correlate with the severity of Gaucher's disease as well as with the response to treatment, ${ }^{84}$ and it has been proposed that the recently discovered 1deoxy-sphingolipids mediate the neurodegeneration of hereditary sensory neuropathy type $1 .{ }^{85}$ Elevated plasma levels of many of the sphingolipids have also been correlated with chronic disease - for example, sphingomyelins with coronary heart disease ${ }^{86}$ and ceramides with type 2 diabetes ${ }^{87}$ and Alzheimer's disease. ${ }^{88}$ Even a very minor species, such as sphinganine, has been useful as a biomarker, since increased maternal serum ratios of sphinganine to sphingosine correlate with the occurrence of neural-tube defects in offspring. ${ }^{89}$ 


\section{CLINICAL PERSPECTIVES ON LIPIDOMICS AND THER APEUTIC MONITORING}

The unexpectedly large number of lipid metabolites present in human plasma establishes a broad platform for the discovery of biomarkers for diseases and efficacy of treatment. Monitoring of specific sterol, cholesterol ester, and TAG molecular species may provide more detailed diagnostic information. Moreover, a recent metaanalysis of genomewide association studies involving more than 100,000 persons identified 95 distinct gene variants associated with lipid traits in plasma that affect blood lipid levels and thus have direct relevance to cardiovascular disease ${ }^{90}$ Combinations of common genetic variants typically contribute to extreme lipid phenotypes, highlighting the multifactorial nature of the metabolic syndrome and cardiovascular disease and the need for comprehensive analyses of lipid metabolites in plasma. Furthermore, the number of distinct human genes and their coded proteins provides a gross underestimate of the number of lipid metabolites because an individual enzyme may catalyze the synthesis of hundreds of distinct lipid species (Fig. 5). These factors, combined with wide-ranging dietary sources of lipids from the plant, animal, and marine kingdoms, as well as the contribution of the gut microbiota, ${ }^{91}$ make it clear that our lipidome is enormous. ${ }^{4}$

The plasma lipidome discussed here is just the tip of the iceberg of what will surely be a vastly expanding library. A recent study followed quantitative changes over time of some 400 lipid molecular species in a macrophage subjected to lipopolysaccharide as a model for infection. ${ }^{92}$ This systems-level view of lipid metabolism reveals important connections between lipid signaling and several biochemical pathways that contribute to innate immune responses in inflammation. The production of eicosanoids is robustly induced by proinflammatory stimuli, and the blocking of underlying signaling events is one of the strategies used by clinicians to manage acute and chronic inflammation. Although plasma eicosanoid concentrations are generally low in the healthy state, circulating eicosanoid levels may increase in disease states as a result of spillover from inflamed tissues. Eicosanoids are also secreted from tissues as part of the catabolic and secretory pathways, and stable metabolites may accumulate in plasma. Isoprostanes derived from nonenzymatic pathways have been found in blood and urine and are currently regarded as sensitive biomarkers of oxidative stress. ${ }^{93}$ In addition, a single-nucleotide polymorphism in the gene encoding prostaglandin $\mathrm{E}$ synthase 2 correlates with increased blood prostaglandin $\mathrm{E}_{2}$ levels, at least in females, and has been associated with type 2 diabetes in several independent cohorts. ${ }^{94,95}$ Not only do eicosanoid levels change, but their patterns of expression may also change, depending on the type of inflammation. Therefore, a complete plasma profile of eicosanoids and their breakdown products should provide an accurate metabolic snapshot.

The development of cancer involves aberrant regulation of multiple cellular processes, including cell proliferation, survival, migration, and invasion and angiogenesis. The actions of a number of phospholipids are concordant with some or all of these hallmarks of cancer, and the composition and level of phospholipids correlate with various types of cancer. Blood is an important reservoir for extracellular S1P, and specific antibodies can potently deplete $\mathrm{S} 1 \mathrm{P}$ in blood and other tissues. Several of these antibodies have reduced tumor progression in animal models and have been formulated for clinical trials of treatment targeting cancer and age-related macular degeneration. ${ }^{96} \mathrm{~S} 1 \mathrm{P}$ also plays an important role in regulating the egress of immune cells from lymphoid tissue into blood. As described above, the S1P analogue fingolimod is approved for the treatment of multiple sclerosis $81,97,98$ and might have a clinical application in other neurodegenerative diseases.

For some neurologic disorders, the initial screening, which often consists of invasive clinical procedures, could be complemented with noninvasive analysis of lipid metabolites in blood. 
For example, the plasma concentrations of glucosylceramide are markedly elevated in patients with symptomatic type 1 Gaucher's disease. ${ }^{84}$ Notably, in one study, all patients had a response to enzymereplacement therapy ${ }^{99}$; within 6 months, plasma glycosylceramide levels were significantly reduced, and these reductions were correlated with clinical responses. Monitoring plasma glucosylceramide levels during therapy is of particular importance because several sphingolipids are effective inhibitors of thrombin generation, 100 which would explain the bleeding tendency in patients with Gaucher's disease. ${ }^{101}$

\section{Conclusions}

Lipid signaling pathways are complex. In the coming years, understanding the integrated lipidomic networks and decoding the coordinately regulated pathways will constitute major goals. The modulation of intracellular and systemic lipid metabolism has enormous therapeutic potential. Recent technological advances have provided the tools for broad lipid profiling as part of the effort to discover biomarkers, which has yielded promising results. ${ }^{102}$ A substantial portion of the human lipidome has now been identified, and methods are available to permit the accurate quantitation of individual lipid molecular species. For the most part, lipidomic studies are performed in specialized laboratories with the use of multiple analytic platforms. Unlike the genome and transcriptome, which can be readily measured with automated array technology, lipids require specific analytic procedures, owing to their diverse structural and physical properties.

The next challenge will be to translate the findings of lipidomic laboratories into medical applications and to introduce these technologies into clinical laboratories. This will require the standardization of sample preparation, the streamlining of analytic procedures, and the establishment of metabolite databases for cross-reference. Despite the work ahead, the use of lipidomics as a diagnostic tool should expand enormously in the coming years as modern medicine evolves to incorporate personalized treatment plans.

\section{Acknowledgments}

We thank our LIPID MAPS colleagues Alex H. Brown, Christopher K. Glass, Alfred H. Merrill, Robert C. Murphy, Christian R.H. Raetz, David W. Russell, Walter A. Shaw, Shankar Subramaniam, Michael S. VanNieuwenhze, Nicholas Winograd, and Joseph L. Witztum for providing valuable scientific input; Dr. M. John Chapman, Pierre and Marie Curie University, for valuable insight and encouragement to write about the plasma lipidome; Yasuyuki Kihara for helping with an earlier version of Figure 5; and Maria Farron for helping with earlier versions of the other figures.

\section{References}

1. Lentner, C., editor. Physical chemistry, composition of blood, hematology, somatometric data. Vol. Vol. 3. Ciba-Geigy; West Caldwell, NJ: 1981. Geigy scientific tables.

2. Wishart DS, Knox C, Guo AC, et al. HMDB: a knowledgebase for the human metabolome. Nucleic Acids Res. 2009; 37:D603-D610. [PubMed: 18953024]

3. Quehenberger O, Armando AM, Brown AH, et al. Lipidomics reveals a remarkable diversity of lipids in human plasma. J Lipid Res. 2010; 51:3299-305. [PubMed: 20671299]

4. Dennis EA. Lipidomics joins the omics evolution. Proc Natl Acad Sci U S A. 2009; 106:2089-90. [PubMed: 19211786]

5. Havel RJ, Eder HA, Bragdon JH. The distribution and chemical composition of ultracentrifugally separated lipoproteins in human serum. J Clin Invest. 1955; 34:1345-53. [PubMed: 13252080]

6. Fahy E, Subramaniam S, Murphy RC, et al. Update of the LIPID MAPS comprehensive classification system for lipids. J Lipid Res. 2009; 50(Suppl):S9-S14. [PubMed: 19098281]

7. Hopkins PN, Heiss G, Ellison RC, et al. Coronary artery disease risk in familial combined hyperlipidemia and familial hypertriglyceridemia: a case-control comparison from the National 
Heart, Lung, and Blood Institute Family Heart Study. Circulation. 2003; 108:519-23. [PubMed: 12847072]

8. Ervin RB. Prevalence of metabolic syndrome among adults 20 years of age and over, by sex, age, race and ethnicity, and body mass index: United States, 2003-2006. Natl Health Stat Rep. 2009; 13:1-7.

9. Pietiläinen KH, Sysi-Aho M, Rissanen A, et al. Acquired obesity is associated with changes in the serum lipidomic profile independent of genetic effects - a monozygotic twin study. PLoS ONE. 2007; 2(2):e218. [PubMed: 17299598]

10. Kotronen A, Velagapudi VR, Yetukuri L, et al. Serum saturated fatty acids containing triacylglycerols are better markers of insulin resistance than total serum triacylglycerol concentrations. Diabetologia. 2009; 52:684-90. [PubMed: 19214471]

11. Graessler J, Schwudke D, Schwarz PE, Herzog R, Shevchenko A, Bornstein SR. Top-down lipidomics reveals ether lipid deficiency in blood plasma of hypertensive patients. PLoS ONE. 2009; 4(7):e6261. [PubMed: 19603071]

12. Sysi-Aho M, Koikkalainen J, Seppänen-Laakso T, et al. Serum lipidomics meets cardiac magnetic resonance imaging: profiling of subjects at risk of dilated cardiomyopathy. PLoS ONE. 2011; 6(1):e15744. [PubMed: 21283746]

13. Pirnay F, Lacroix M, Mosora F, Luyckx A, Lefebvre P. Effect of glucose ingestion on energy substrate utilization during prolonged muscular exercise. Eur J Appl Physiol Occup Physiol. 1977; 36:247-54. [PubMed: 902638]

14. Department of Agriculture, Center for Nutrition Policy and Promotion. Is total fat consumption really decreasing?. Nutrition Insights. Apr. 1998 http://www.cnpp.usda.gov/Publications/NutritionInsights/insight5.pdf

15. Ogden, CL.; Carroll, MD.; McDowell, MA.; Flegal, KM. Obesity among adults in the United States - no statistically significant chance since 2003-2004. National Center for Health Statistics Data Brief. Nov. 2007 http://www.cdc.gov/nchs/data/databriefs/db01.pdf

16. Conner WE, Lin DS, Colvis C. Differential mobilization of fatty acids from adipose tissue. J Lipid Res. 1996; 37:290-8. [PubMed: 9026527]

17. DeLany JP, Windhauser MM, Champagne CM, Bray GA. Differential oxidation of individual dietary fatty acids in humans. Am J Clin Nutr. 2000; 72:905-11. [PubMed: 11010930]

18. Reaven GM, Hollenbeck C, Jeng CY, Wu MS, Chen YD. Measurement of plasma glucose, free fatty acid, lactate, and insulin for $24 \mathrm{~h}$ in patients with NIDDM. Diabetes. 1988; 37:1020-4. [PubMed: 3292322]

19. Boden G. Fatty acid-induced inflammation and insulin resistance in skeletal muscle and liver. Curr Diab Rep. 2006; 6:177-81. [PubMed: 16898568]

20. Donnelly KL, Smith CI, Schwarzenberg SJ, Jessurun J, Boldt MD, Parks EJ. Sources of fatty acids stored in liver and secreted via lipoproteins in patients with nonalcoholic fatty liver disease. J Clin Invest. 2005; 115:1343-51. [PubMed: 15864352]

21. Mulhall BP, Ong JP, Younossi ZM. Non-alcoholic fatty liver disease: an overview. J Gastroenterol Hepatol. 2002; 17:1136-43. [PubMed: 12453271]

22. Santomauro AT, Boden G, Silva ME, et al. Overnight lowering of free fatty acids with Acipimox improves insulin resistance and glucose tolerance in obese diabetic and nondiabetic subjects. Diabetes. 1999; 48:1836-41. [PubMed: 10480616]

23. Ginsberg HN, Zhang YL, Hernandez-Ono A. Metabolic syndrome: focus on dyslipidemia. Obesity (Silver Spring). 2006; 14(Suppl 1):41S-49S. [PubMed: 16642962]

24. Charles MA, Eschwège E, Thibult N, et al. The role of non-esterified fatty acids in the deterioration of glucose tolerance in Caucasian subjects: results of the Paris Prospective Study. Diabetologia. 1997; 40:1101-6. [PubMed: 9300248]

25. Riccardi G, Giacco R, Rivellese AA. Dietary fat, insulin sensitivity and the metabolic syndrome. Clin Nutr. 2004; 23:447-56. [PubMed: 15297079]

26. Matsuzaka T, Shimano H, Yahagi N, et al. Crucial role of a long-chain fatty acid elongase, Elovl6, in obesity-induced insulin resistance. Nat Med. 2007; 13:1193-202. [PubMed: 17906635] 
27. Maeda K, Cao H, Kono K, et al. Adipocyte/macrophage fatty acid binding proteins control integrated metabolic responses in obesity and diabetes. Cell Metab. 2005; 1:107-19. [PubMed: 16054052]

28. Cao H, Gerhold K, Mayers JR, Wiest MM, Watkins SM, Hotamisligil GS. Identification of a lipokine, a lipid hormone linking adipose tissue to systemic metabolism. Cell. 2008; 134:933-44. [PubMed: 18805087]

29. Dimopoulos N, Watson M, Sakamoto K, Hundal HS. Differential effects of palmitate and palmitoleate on insulin action and glucose utilization in rat L6 skeletal muscle cells. Biochem J. 2006; 399:473-81. [PubMed: 16822230]

30. Stefan N, Kantartzis K, Celebi N, et al. Circulating palmitoleate strongly and independently predicts insulin sensitivity in humans. Diabetes Care. 2010; 33:405-7. [PubMed: 19889804]

31. Mozaffarian D, Cao H, King IB, et al. Circulating palmitoleic acid and risk of metabolic abnormalities and new-onset diabetes. Am J Clin Nutr. 2010; 92:1350-8. [PubMed: 20943795]

32. Trans-palmitoleic acid, metabolic risk factors, and new-onset diabetes in U.S. adults: a cohort study. Ann Intern Med. 2010; 153:790-9. Idem. [PubMed: 21173413]

33. Pereira MA, Jacobs DR Jr, Van Horn L, Slattery ML, Kartashov AI, Ludwig DS. Dairy consumption, obesity, and the insulin resistance syndrome in young adults: the CARDIA Study. JAMA. 2002; 287:2081-9. [PubMed: 11966382]

34. Leaf A. Plasma nonesterified fatty acid concentration as a risk factor for sudden cardiac death: the Paris Prospective Study. Circulation. 2001; 104:744-5. [PubMed: 11502694]

35. Legaspi A, Jeevanandam M, Starnes HF Jr, Brennan MF. Whole body lipid and energy metabolism in the cancer patient. Metabolism. 1987; 36:958-63. [PubMed: 3657515]

36. Kleinfeld AM, Okada C. Free fatty acid release from human breast cancer tissue inhibits cytotoxic T-lymphocyte-mediated killing. J Lipid Res. 2005; 46:1983-90. [PubMed: 15961785]

37. McLennan PL. Relative effects of dietary saturated, monounsaturated, and polyunsaturated fatty acids on cardiac arrhythmias in rats. Am J Clin Nutr. 1993; 57:207-12. [PubMed: 8424390]

38. Burr ML, Fehily AM, Gilbert JF, et al. Effects of changes in fat, fish, and fibre intakes on death and myocardial reinfarction: Diet and Reinfarction Trial (DART). Lancet. 1989; 2:757-61. [PubMed: 2571009]

39. Buczynski MW, Dumlao DS, Dennis EA. Thematic review series: proteomics — an integrated omics analysis of eicosanoid biology. J Lipid Res. 2009; 50:1015-38. [PubMed: 19244215] J Lipid Res. 2009; 50:1505. Erratum.

40. Kris-Etherton PM, Harris WS, Appel LJ. Fish consumption, fish oil, omega-3 fatty acids, and cardiovascular disease. Circulation. 2002; 106:2747-57. [PubMed: 12438303] Circulation. 2003; 107:512. Erratum.

41. Itoh Y, Kawamata Y, Harada M, et al. Free fatty acids regulate insulin secretion from pancreatic beta cells through GPR40. Nature. 2003; 422:173-6. [PubMed: 12629551]

42. Oh DY, Talukdar S, Bae EJ, et al. GPR120 is an omega-3 fatty acid receptor mediating potent antiinflammatory and insulinsensitizing effects. Cell. 2010; 142:687-98. [PubMed: 20813258]

43. Schaefer EJ, Bongard V, Beiser AS, et al. Plasma phosphatidylcholine docosahexaenoic acid content and risk of dementia and Alzheimer disease: the Framingham Heart Study. Arch Neurol. 2006; 63:1545-50. [PubMed: 17101822]

44. Barberger-Gateau P, Raffaitin C, Letenneur L, et al. Dietary patterns and risk of dementia: the Three-City cohort study. Neurology. 2007; 69:1921-30. [PubMed: 17998483]

45. Maes M, Smith R, Christophe A, Cosyns P, Desnyder R, Meltzer H. Fatty acid composition in major depression: decreased omega 3 fractions in cholesteryl esters and increased C20: 4 omega 6/ C20:5 omega 3 ratio in cholesteryl esters and phospholipids. J Affect Disord. 1996; 38:35-46. [PubMed: 8735157]

46. Stoll AL, Severus WE, Freeman MP, et al. Omega 3 fatty acids in bipolar disorder: a preliminary double-blind, placebocontrolled trial. Arch Gen Psychiatry. 1999; 56:407-12. [PubMed: 10232294]

47. Orešič M, Tang J, Seppänen-Laakso T, et al. Metabolome in schizophrenia and other psychotic disorders: a general population-based study. Genome Med. 2011; 3:19. [PubMed: 21429189] 
48. Executive summary of the Third Report of the National Cholesterol Education Program (NCEP) Expert Panel on Detection, Evaluation, and Treatment of High Blood Cholesterol in Adults (Adult Treatment Panel III). JAMA. 2001; 285:2486-97. [PubMed: 11368702]

49. Bhattacharyya AK, Connor WE. Betasitosterolemia and xanthomatosis: a newly described lipid storage disease in two sisters. J Clin Invest. 1974; 53:1033-43. [PubMed: 4360855]

50. Berge KE, Tian H, Graf GA, et al. Accumulation of dietary cholesterol in sitosterolemia caused by mutations in adjacent ABC transporters. Science. 2000; 290:1771-5. [PubMed: 11099417]

51. Rader DJ, Cohen J, Hobbs HH. Monogenic hypercholesterolemia: new insights in pathogenesis and treatment. J Clin Invest. 2003; 111:1795-803. [PubMed: 12813012]

52. Watts GF, Mitchell WD, Bending JJ, Reshef A, Leitersdorf E. Cerebrotendinous xanthomatosis: a family study of sterol 27-hydroxylase mutations and pharmacotherapy. QJM. 1996; 89:55-63. [PubMed: 8730343]

53. Björkhem I, Miettinen T, Reihnér E, Ewerth S, Angelin B, Einarsson K. Correlation between serum levels of some cholesterol precursors and activity of HMGCoA reductase in human liver. $\mathrm{J}$ Lipid Res. 1987; 28:1137-43. [PubMed: 3681138]

54. Bauman DR, Bitmansour AD, McDonald JG, Thompson BM, Liang G, Russell DW. 25Hydroxycholesterol secreted by macrophages in response to Toll-like receptor activation suppresses immunoglobulin A production. Proc Natl Acad Sci U S A. 2009; 106:16764-9. [PubMed: 19805370]

55. Negendank W. Studies of human tumors by MRS: a review. NMR Biomed. 1992; 5:303-24. [PubMed: 1333263]

56. Ackerstaff E, Glunde K, Bhujwalla ZM. Choline phospholipid metabolism: a target in cancer cells? J Cell Biochem. 2003; 90:525-33. [PubMed: 14523987]

57. Nakagami K, Uchida T, Ohwada S, et al. Increased choline kinase activity and elevated phosphocholine levels in human colon cancer. Jpn J Cancer Res. 1999; 90:419-24. [PubMed: 10363580]

58. Ramírez de Molina A, Gutiérrez R, Ramos MA, et al. Increased choline kinase activity in human breast carcinomas: clinical evidence for a potential novel antitumor strategy. Oncogene. 2002; 21:4317-22. [PubMed: 12082619]

59. Iorio E, Ricci A, Bagnoli M, et al. Activation of phosphatidylcholine cycle enzymes in human epithelial ovarian cancer cells. Cancer Res. 2010; 70:2126-35. [PubMed: 20179205]

60. Ramírez de Molina A, Sarmentero-Estrada J, Belda-Iniesta C, et al. Expres-sion of choline kinase alpha to predict outcome in patients with early-stage non-small-cell lung cancer: a retrospective study. Lancet Oncol. 2007; 8:889-97. [PubMed: 17851129]

61. Hernando E, Sarmentero-Estrada J, Koppie T, et al. A critical role for choline kinase-alpha in the aggressiveness of bladder carcinomas. Oncogene. 2009; 28:2425-35. [PubMed: 19448670]

62. Jagannathan NR, Kumar M, Seenu V, et al. Evaluation of total choline from invivo volume localized proton MR spectroscopy and its response to neoadjuvant chemotherapy in locally advanced breast cancer. Br J Cancer. 2001; 84:1016-22. [PubMed: 11308247]

63. Griffiths JR, Tate AR, Howe FA, Stubbs M. Magnetic resonance spectroscopy of cancerpracticalities of multi-centre trials and early results in non-Hodgkin's lymphoma. Eur J Cancer. 2002; 38:2085-93. [PubMed: 12387834]

64. Murphy PS, Viviers L, Abson C, et al. Monitoring temozolomide treatment of low-grade glioma with proton magnetic resonance spectroscopy. Br J Cancer. 2004; 90:781-6. [PubMed: 14970853]

65. Foster DA. Phosphatidic acid signaling to mTOR: signals for the survival of human cancer cells. Biochim Biophys Acta. 2009; 1791:949-55. [PubMed: 19264150]

66. Schaloske RH, Dennis EA. The phospholipase A2 superfamily and its group numbering system. Biochim Biophys Acta. 2006; 1761:1246-59. [PubMed: 16973413]

67. Tokumura A, Majima E, Kariya Y, et al. Identification of human plasma lysophospholipase D, a lysophosphatidic acid-producing enzyme, as autotaxin, a multifunctional phosphodiesterase. J Biol Chem. 2002; 277:39436-42. [PubMed: 12176993]

68. Moolenaar WH, van Meeteren LA, Giepmans BN. The ins and outs of lysophosphatidic acid signaling. Bioessays. 2004; 26:870-81. [PubMed: 15273989] 
69. Mills GB, Moolenaar WH. The emerging role of lysophosphatidic acid in cancer. Nat Rev Cancer. 2003; 3:582-91. [PubMed: 12894246]

70. Xu Y, Gaudette DC, Boynton JD, et al. Characterization of an ovarian cancer activating factor in ascites from ovarian cancer patients. Clin Cancer Res. 1995; 1:1223-32. [PubMed: 9815916]

71. Mills GB, May C, Hill M, Campbell S, Shaw P, Marks A. Ascitic fluid from human ovarian cancer patients contains growth factors necessary for intraperitoneal growth of human ovarian adenocarcinoma cells. J Clin Invest. 1990; 86:851-5. [PubMed: 2394835]

72. Xu Y, Shen Z, Wiper DW, et al. Lysophosphatidic acid as a potential biomarker for ovarian and other gynecologic cancers. JAMA. 1998; 280:719-23. [PubMed: 9728644]

73. Baker DL, Morrison P, Miller B, et al. Plasma lysophosphatidic acid concentration and ovarian cancer. JAMA. 2002; 287:3081-2. [PubMed: 12069669]

74. Sutphen R, Xu Y, Wilbanks GD, et al. Lysophospholipids are potential biomarkers of ovarian cancer. Cancer Epidemiol Biomarkers Prev. 2004; 13:1185-91. [PubMed: 15247129]

75. Pozlep B, Meleh M, Kobal B, et al. Use of lysophosphatidic acid in the management of benign and malignant ovarian tumors. Eur J Gynaecol Oncol. 2007; 28:394-9. [PubMed: 17966220]

76. Sedláková I, Vávrová J, Tošner J, Hanousek L. Lysophosphatidic acid (LPA) — a perspective marker in ovarian cancer. Tumour Biol. 2011; 32:311-6. [PubMed: 21061112]

77. Bese T, Barbaros M, Baykara E, et al. Comparison of total plasma lysophosphatidic acid and serum CA-125 as a tumor marker in the diagnosis and follow-up of patients with epithelial ovarian cancer. J Gynecol Oncol. 2010; 21:248-54. [PubMed: 21278887]

78. Lee MJ, Van Brocklyn JR, Thangada S, et al. Sphingosine-1-phosphate as a ligand for the G protein-coupled receptor EDG-1. Science. 1998; 279:1552-5. [PubMed: 9488656]

79. Brinkmann V. Sphingosine 1-phosphate receptors in health and disease: mechanistic insights from gene deletion studies and reverse pharmacology. Pharmacol Ther. 2007; 115:84-105. [PubMed: 17561264]

80. Matloubian M, Lo CG, Cinamon G, et al. Lymphocyte egress from thymus and peripheral lymphoid organs is dependent on S1P receptor 1. Nature. 2004; 427:355-60. [PubMed: 14737169]

81. Brinkmann V, Billich A, Baumruker T, et al. Fingolimod (FTY720): discovery and development of an oral drug to treat multiple sclerosis. Nat Rev Drug Discov. 2010; 9:883-97. [PubMed: 21031003]

82. Hammad SM, Pierce JS, Soodavar F, et al. Blood sphingolipidomics in healthy humans: impact of sample collection methodology. J Lipid Res. 2010; 51:3074-87. [PubMed: 20660127]

83. Scherer M, Böttcher A, Schmitz G, Liebisch G. Sphingolipid profiling of human plasma and FPLC-separated lipoprotein fractions by hydrophilic interaction chromatography tandem mass spectrometry. Biochim Biophys Acta. 2011; 1811:68-75. [PubMed: 21081176]

84. Groener JE, Poorthuis BJ, Kuiper S, Hollak CE, Aerts JM. Plasma glucosylceramide and ceramide in type 1 Gaucher disease patients: correlations with disease severity and response to therapeutic intervention. Biochim Biophys Acta. 2008; 1781:72-8. [PubMed: 18155675]

85. Penno A, Reilly MM, Houlden H, et al. Hereditary sensory neuropathy type 1 is caused by the accumulation of two neurotoxic sphingolipids. J Biol Chem. 2010; 285:11178-87. [PubMed: 20097765]

86. Yeboah J, McNamara C, Jiang XC, et al. Association of plasma sphingomyelin levels and incident coronary heart disease events in an adult population: Multi-Ethnic Study of Atherosclerosis. Arterioscler Thromb Vasc Biol. 2010; 30:628-33. [PubMed: 20032291]

87. Haus JM, Kashyap SR, Kasumov T, et al. Plasma ceramides are elevated in obese subjects with type 2 diabetes and correlate with the severity of insulin resistance. Diabetes. 2009; 58:337-43. [PubMed: 19008343]

88. Ewers M, Mielke MM, Hampel H. Blood-based biomarkers of microvascular pathology in Alzheimer's disease. Exp Gerontol. 2010; 45:75-9. [PubMed: 19782124]

89. Missmer SA, Suarez L, Felkner M, et al. Exposure to fumonisins and the occurrence of neural tube defects along the Texas-Mexico border. Environ Health Perspect. 2006; 114:237-41. [PubMed: 16451860]

90. Teslovich TM, Musunuru K, Smith AV, et al. Biological, clinical and population relevance of 95 loci for blood lipids. Nature. 2010; 466:707-13. [PubMed: 20686565] 
91. Ley RE, Hamady M, Lozupone C, et al. Evolution of mammals and their gut microbes. Science. 2008; 320:1647-51. [PubMed: 18497261] Science. 2008; 322:1188. Erratum.

92. Dennis EA, Deems RA, Harkewicz R, et al. A mouse macrophage lipidome. J Biol Chem. 2010; 285:39976-85. [PubMed: 20923771]

93. Roberts LJ, Morrow JD. Measurement of F(2)-isoprostanes as an index of oxidative stress in vivo. Free Radic Biol Med. 2000; 28:505-13. [PubMed: 10719231]

94. Nitz I, Fisher E, Grallert H, et al. Association of prostaglandin E synthase 2 (PTGES2) Arg298His polymorphism with type 2 diabetes in two German study populations. J Clin Endocrinol Metab. 2007; 92:3183-8. [PubMed: 17566096]

95. Boomgaarden I, Bosy-Westphal A, Müller MJ, Doring F. Influence of a type 2 diabetes associated prostaglandin E synthase 2 polymorphism on blood prostaglandin E2 levels. Prostaglandins Leukot Essent Fatty Acids. 2009; 80:185-8. [PubMed: 19268562]

96. O’Brien N, Jones ST, Williams DG, et al. Production and characterization of monoclonal antisphingosine-1-phosphate anti-bodies. J Lipid Res. 2009; 50:2245-57. [PubMed: 19509417]

97. Kappos L, Radue EW, O’Connor P, et al. A placebo-controlled trial of oral fingolimod in relapsing multiple sclerosis. N Engl J Med. 2010; 362:387-401. [PubMed: 20089952]

98. Cohen JA, Barkhof F, Comi G, et al. Oral fingolimod or intramuscular interferon for relapsing multiple sclerosis. N Engl J Med. 2010; 362:402-15. [PubMed: 20089954]

99. de Fost M, Hollak CE, Groener JE, et al. Superior effects of high-dose enzyme replacement therapy in type 1 Gaucher disease on bone marrow involvement and chito-triosidase levels: a 2center retrospective analysis. Blood. 2006; 108:830-5. [PubMed: 16527890]

100. Deguchi H, Yegneswaran S, Griffin JH. Sphingolipids as bioactive regulators of thrombin generation. J Biol Chem. 2004; 279:12036-42. [PubMed: 14722105]

101. Hollak CE, Levi M, Berends F, Aerts JM, van Oers MH. Coagulation abnormalities in type 1 Gaucher disease are due to low-grade activation and can be partly restored by enzyme supplementation therapy. Br J Haematol. 1997; 96:470-6. [PubMed: 9054650]

102. Harkewicz R, Dennis EA. Applications of mass spectrometry to lipids and membranes. Annu Rev Biochem. 2011; 80:301-25. [PubMed: 21469951] 


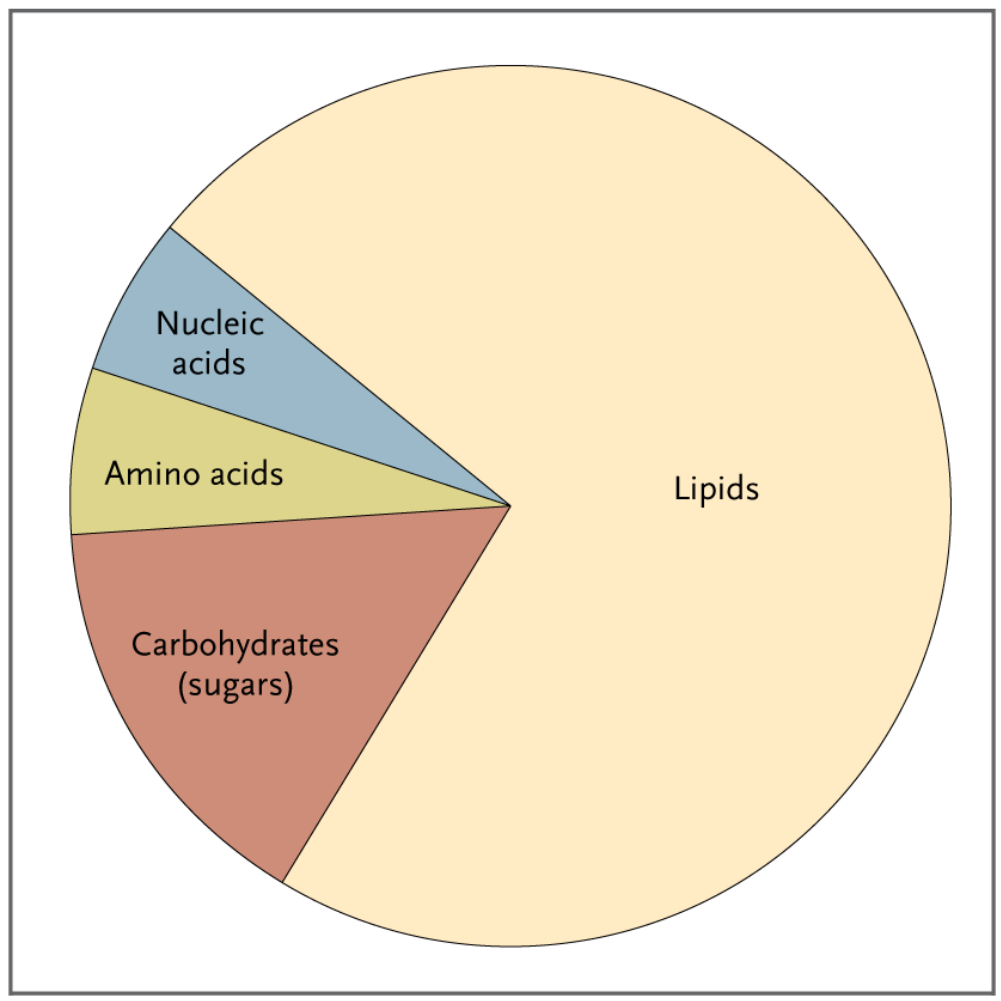

Figure 1. Relative Distribution of Biologic Molecules in Human Plasma

Amino acids and nucleic acids are shown without consideration of the contribution of proteins and DNA or RNA. The relative distribution is based on weight (grams per deciliter). Data were compiled from Lentner, ${ }^{1}$ Wishart et al., ${ }^{2}$ and Quehenberger et al. ${ }^{3}$ 


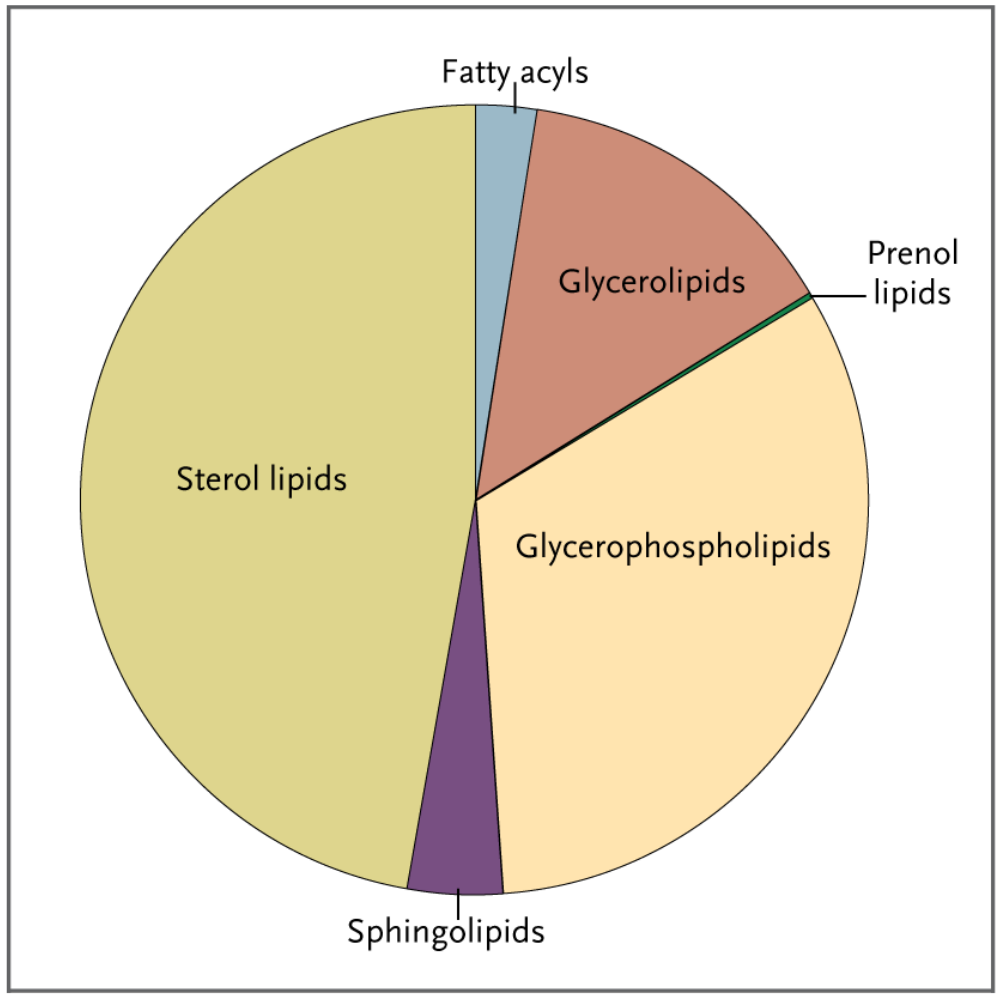

Figure 2. Relative Distribution of Lipids in Human Plasma

Lipidomic analysis has identified, characterized, and quantified almost 600 lipid molecular species in human plasma. ${ }^{3}$ The relative distribution in each category is given on a molar basis. The nomenclature of the lipid categories conforms to the recently developed LIPID MAPS classification system. ${ }^{6}$ 


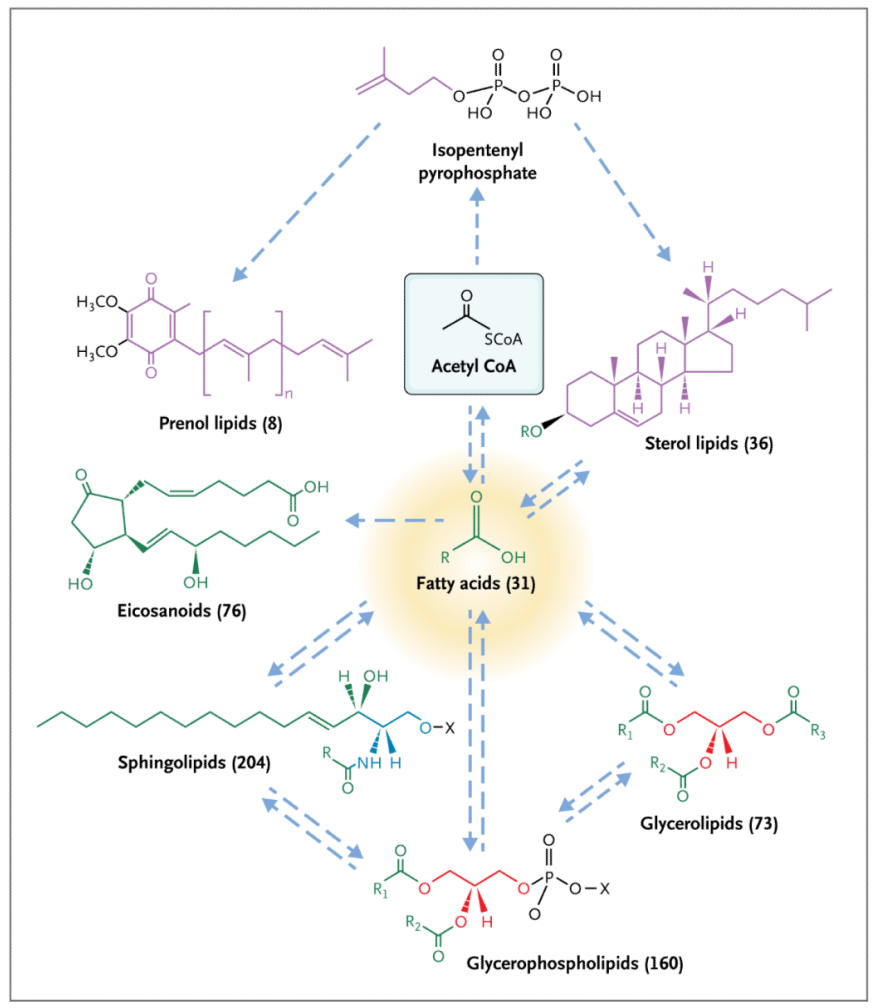

Figure 3. Diversity of Human Plasma Lipids

Relationships among the major mammalian lipid categories are shown by means of representative molecules from each class as examples. The diagram starts with the 2-carbon precursor acetyl coenzyme A (CoA), which is the building block for the biosynthesis of fatty acids. (SCoA indicates the thioester bond between CoA and acetic acid.) Fatty acids, in turn, become part of complex lipids - namely, glycerolipids, glycerophospholipids, sphingolipids, and sterol lipids (as steryl esters). Some fatty acids are converted to eicosanoids. A second major biosynthetic route from acetyl CoA generates the 5-carbon isoprene precursor isopentenyl pyrophosphate, which provides the building block for the prenol and sterol lipids. (The $n$ indicates the variable number of isoprene units.) Fatty acylderived substituents are shown in green, isoprene-derived atoms are shown in purple, the glycerol backbone is shown in red, and the serinederived backbone is shown in blue. Arrows indicate multistep transformations among the major lipid categories, starting with acetyl CoA. Values in parentheses indicate the number of distinct analytes within each lipid category that were quantified by means of mass spectrometry in the human plasma lipidome. ${ }^{3}$ 


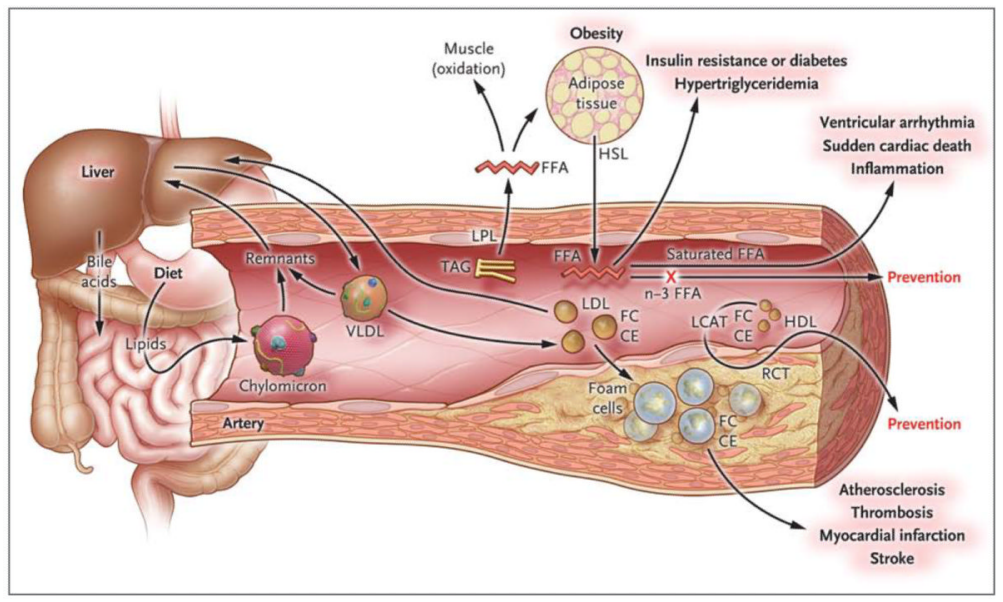

Figure 4. Plasma Lipids in the Metabolic Syndrome

Abnormal levels of plasma lipids and lipoproteins are important risk factors for metabolic and cardiovascular diseases and are targets for therapeutic intervention. Cells need cholesterol and triacylglycerol (TAG) derived from dietary sources and the liver for membrane synthesis and energy. These lipids circulate in the blood as lipoprotein particles, including chylomicrons, very-low-density lipoprotein (VLDL), lowdensity lipoprotein (LDL), and high-density lipoprotein (HDL). In circulating chylomicrons and VLDL, TAG undergoes hydrolysis, catalyzed by lipoprotein lipase (LPL), to generate a pool of free fatty acids (FFAs) that is used as an energy source in tissues, including muscle. Excess FFAs are stored in adipocytes in the form of TAGs. Such caloric abundance leads to an unopposed expansion of adipose tissue and, ultimately, to obesity and associated metabolic complications characterized by insulin resistance and diabetes. Stored TAG in adipocytes undergoes lipolysis on demand as a result of hormone-sensitive lipase (HSL), leading to an energy-balanced level of FFAs in plasma. In insulin resistance, adipocytes exhibit a high rate of lipolysis and are highly responsive to fat-mobilizing enzymes but respond poorly to lipolysisrestraining insulin. Furthermore, insulin resistance depresses adipocyte LPL activity; however, adipocytes from obese humans use compensatory mechanisms that increase the capacity for FFA transport and uptake. In combination with increased lipolysis, this process generates abnormally high plasma levels of FFAs, allowing their increased uptake into hepatocytes in excess of metabolic requirements, which leads to storage as TAG and results in hepatic steatosis and inflammation. Some TAGs are exported as VLDL, contributing to hypertriglyceridemia. Trans-palmitoleic acid may oppose some of these effects and may stimulate insulin sensitivity in muscle and liver. In general, saturated FFAs promote cardiac disorders and systemic inflammation, whereas n-3 FFAs prevent these effects. The contribution of LDL-derived cholesterol, in both its free form (FC) and its esterified form (CE), to the development of cardiovascular disease has been well described. HDL helps remove excess FC by reverse cholesterol transport (RCT), with the formation of $\mathrm{CE}$ by lecithin cholesterol acyltransferase (LCAT), and subsequent uptake of the CE by the liver. High levels of HDL are correlated with low cardiovascular risk. 


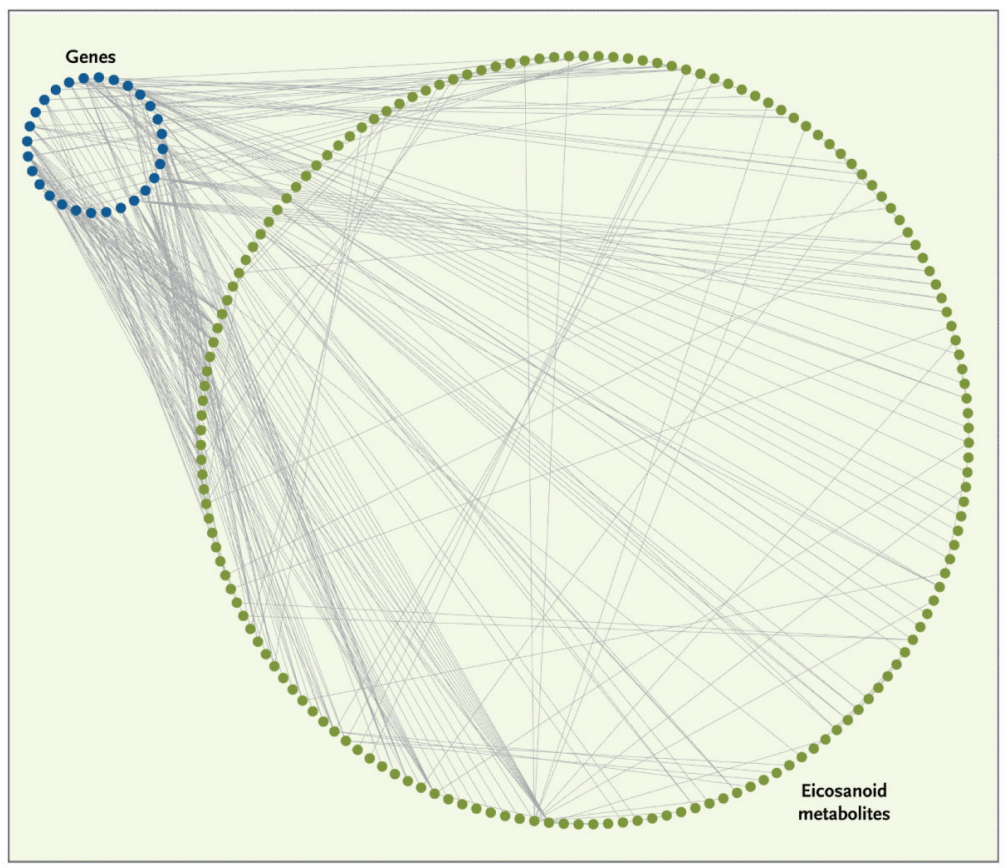

Figure 5. Eicosanoid Metabolic Network

The number of genes and proteins in a biosynthetic pathway does not accurately reflect the enormous diversity of the lipidome. For example, in the eicosanoid biosynthetic pathways, 28 known genes and their corresponding enzymatic gene products (green dots) are responsible for the production of more than 150 bioactive lipids (yellow dots) derived from dihomo-gamma-linolenic acid (20:3), arachidonic acid (20:4), eicosapentaenoic acid (20:5), and docosahexaenoic acid (22:6), ${ }^{39}$ of which 76 have been detected in normal human plasma. ${ }^{3}$ (Fatty acids are defined by the ratio of the number of carbon atoms to the number of double bonds.) A similar discrepancy between the small numbers of genes and the correspondingly larger number of individual lipid species is observed with all other lipid categories, and overlapping enzymatic activities explain the fact that a relatively small set of enzymes generates a vast number of distinct lipid species with defined molecular structures and unique biologic functions. 